\title{
Sperm Quality and Deoxyribonucleic Acid Fragmentation after 5 and 10 min Centrifugation with Swim-Up Processing Technique: A Prospective Cohort Study
}

\author{
Binarwan Halim ${ }^{1,2}$, Jesselyn Angellee ${ }^{1 *}$ iD, Hilma Putri Lubis ${ }^{1,2}$, Bob Bachsinar $^{1}$ \\ ${ }^{1}$ Halim Fertility Center, Stella Maris Women's and Children's Hospital, Medan, Indonesia; ${ }^{2}$ Department of Obstetrics and \\ Gynecology, Division of Reproductive, Endocrinology and Infertility, Faculty of Medicine, Universitas Sumatera Utara, Haji \\ Adam Malik General Hospital, Medan, Indonesia
}

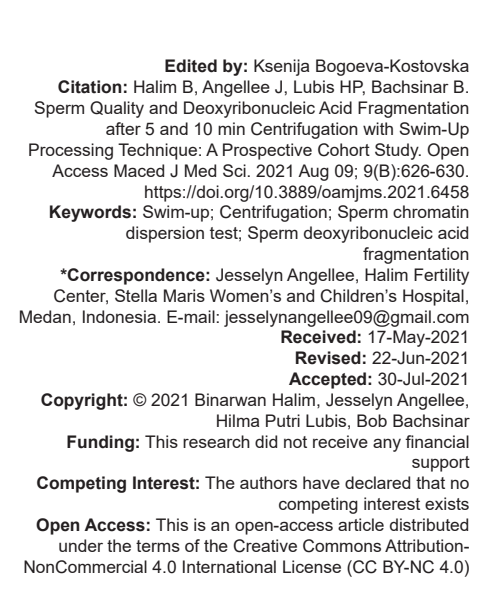

\begin{abstract}
AIM: This study aimed to assess the difference in sperm concentration, total motility, progressive motility, and deoxyribonucleic acid (DNA) fragmentation index (DFI) before and after processing with 5 and 10 min swim-up (SU).

METHODS: Fifty patients who met the study inclusion criteria from June 2020 to October 2020 were subjected to routine semen and sperm DNA fragmentation analysis. Each of the samples was then divided into three tubes, one as control and the others were processed using the SU method with 5 and 10 min centrifugation time, respectively. After being processed, the samples were subjected again to routine semen and sperm DNA fragmentation analysis. The results were being compared among three groups.

RESULTS: The sperm concentration after 5 and $10 \mathrm{~min}$ SU (27.78-39.79 and 35.36-51.09, respectively; $p<0.05)$ was significantly higher compared to fresh semen (24.85-32.33). The total motility before and after 5 and $10 \mathrm{~min}$ SU were 43.78-51.08, 97.66-98.20, and 97.86-98.20, respectively. The progressive motility after 5 and 10 min SU (0-41 and $0-54$, respectively) was significantly higher than fresh semen $(0-24 ; p<0.05)$. The DFI was significantly better after 5 min SU (3.82-6.98) compared to fresh semen and after 10 min SU (13.48-19.04 and 1-25, respectively; $\mathrm{p}<0.05)$.

CONCLUSION: Prolonged centrifugation time may yield a higher number of sperm concentration and motility, but it may also lead to a higher DFI. Hence, a shorter centrifugation time should be used for a better semen quality intended for assisted reproductive technologies.
\end{abstract}

\section{Introduction}

Infertility is a condition where couples who have had regular unprotected sexual intercourses (2-3 times a week) are unable to conceive and this condition lasts for at least 12 months. Infertility is a global problem as it can be found in at least 48.5 million couples worldwide. Male infertility accounts for $30-40 \%$ of infertility cases, most of which are known to be the result of damage to the deoxyribonucleic acid (DNA) of the sperm [1]. In general, sperm is very sensitive to oxidative stress which is known to disrupt the function of their membranes, decrease the capacitation ability, impair the DNA, and may even result in cell death [2]. Several studies have shown that sperm DNA fragmentation index (DFI) can be used to predict male fertility status, as it has better diagnostic and prognostic values than routine sperm analysis. So far, the integrity of DNA or chromatin in sperm has been the most studied molecular problem and fragmentation of the DNA has been linked to decreased fertility rate, impaired pre-implantation development, increased incidence of recurrent pregnancy loss, and increased infant mortality [3], [4]

One of the many factors that may cause sperm DNA damage is the sperm preparation technique. Normally, the process of separating motile from immotile sperm, debris, seminal plasma, and leukocytes takes place in the female reproductive tract through an active transfer process with the help of cervical mucus [2]. In the context of in vitro fertilization (IVF), the preparation process is aimed at obtaining sperm with good viability and high motility, as well as to reduce the percentage of DNA damage and sperm with abnormal morphology. There are several methods of sperm preparation that is commonly used, one of which is swim-up (SU) [5], [6]. In general, sperm preparation techniques involve centrifugation to obtain pellets, but this process is known to cause damage to sperm, which in some cases may also lead to pregnancy defects or failure [7]. Such condition may occur due to the risk that sperm preparation methods are thought to induce uncontrolled production of reactive oxygen species (ROS). If the production of ROS exceeds the antioxidant capacity of the sperm, DNA integrity will 
be damaged. The sperm membrane contains a high amount of polyunsaturated fatty acids, which is why the interaction between ROS and the membrane will form fat electrophiles that are associated with oxidative stress and ultimately cause fat peroxidation. The peroxidation of fat in the sperm membrane may cause DNA damage or fragmentation [5].

In this study, we aimed to assess whether there was a difference in the quality and DFI among fresh semen and semen processed using the 5 and $10 \mathrm{~min}$ SU method.

\section{Methods}

\section{Patients}

This is a prospective cohort study of male patients who will undergo routine semen and sperm DNA fragmentation analysis at Halim Fertility Center, Stella Maris Women's and Children's Hospital, Medan, Indonesia, from June 2020 to October 2020. Sampling was carried out using the consecutive sampling method, where samples were selected based on inclusion and exclusion criteria. The inclusion criteria of this study were male patients who were willing to participate in the study, aged 25-45 years, underwent sexual abstinence for at least 2 days and a maximum of 7 days before the analysis, had a semen volume of at least $1.5 \mathrm{~mL}$, minimum sperm concentration of $5 \times 10^{6}$ per $\mathrm{mL}$, no history of fever, genital infection, or consuming drugs that can impair the sperm in the past 3 months, no history of infectious diseases (hepatitis $B$, hepatitis $C$, and HIV), retrograde ejaculation, cryptorchidism, and testicular cancer. Meanwhile, the exclusion criteria for this study were male patients who were not willing to participate in the study and defective samples. Of all who came, 50 patients met the study criteria and were recorded for their name, wife's name, medical record number, height, weight, history of smoking, history of alcohol consumed, history of drugs consumed, history of systemic diseases (diabetes, kidney failure, hyperthyroid, and hypothyroid), and duration of sexual abstinence.

\section{Semen collection and analysis}

Fifty patients who met the study inclusion criteria were asked to accommodate the sample in a wide mouth, sterile plastic, or glass container. After being liquefied for approximately 30-60 min at room temperature, the samples were subjected to routine semen analysis based on the World Health Organization (WHO) 2010 standards to assess the volume, $\mathrm{pH}$, viscosity, liquefaction, sperm concentration, motility, and morphology.

\section{Semen preparation using the SU method}

The previously analyzed semen samples were mixed using $1 \mathrm{~mL}$ of G-MOPS ${ }^{\mathrm{TM}}$ PLUS medium containing human serum albumin and gentamicin. The samples were transferred into three sterile $5 \mathrm{~mL}$ falcon tubes, each labeled A, B, and C. Tube A contained unprocessed semen samples, while tube $B$ and tube $C$ were centrifuged at a speed of $1400 \mathrm{rpm}$, each for 5 and $10 \mathrm{~min}$. The supernatant from both tubes was removed afterward, leaving the sperm pellets at the bottom of the tube. The pellets were then overlayed with $0.5-1 \mathrm{~mL}$ of G-MOPS ${ }^{\mathrm{TM}}$ PLUS medium and inclined at $45^{\circ}$ for $45 \mathrm{~min}$ at $37^{\circ} \mathrm{C}$. After $45 \mathrm{~min}$, the top layer of the sample was transferred to a new sterile $5 \mathrm{~mL}$ falcon tube and then a routine semen analysis was carried out for each tube B and C according to the WHO 2010 standards to assess the sperm concentration and motility.

\section{Sperm DNA fragmentation assessment}

We used the SpermFunc ${ }^{\circledR}$ DNAf kit (BRED Life Science Technology Inc., China) to evaluate the DNA intactness in the sperm. The vial containing agarose gel was put in an $80^{\circ} \mathrm{C}$ water bath for at least 20 min for the gel to dissolve completely. Around $60 \mu \mathrm{L}$ of each semen sample (before processing, after 5 min centrifugation $\mathrm{SU}$, and after $10 \mathrm{~min}$ centrifugation SU) was added and mixed thoroughly to three different vials containing dissolved agarose gel, then $30 \mu \mathrm{L}$ of the suspension was dispensed to the pre-coated slide and covered with a cover slip. The slide was then kept at $2-8^{\circ} \mathrm{C}$ for $5 \mathrm{~min}$. After the suspension had congealed, the cover was carefully removed and the pre-coated slide was then soaked into the denaturation solution for $7 \mathrm{~min}$ at room temperature. After clearing the remaining fluid on the back and side of the slide, it was soaked in the lysis solution for another $25 \mathrm{~min}$ at room temperature. The slide was then washed with distilled water for $5 \mathrm{~min}$, dried, and dipped into a series of ethanol, $70 \%, 90 \%$, and $100 \%$, each for $2 \mathrm{~min}$. The slide was dried again before stained in Wright's stain for $15 \mathrm{~min}$. After rinsed and dried, the slide was observed under the optical microscope with $40 x$ field lens to count the sperm with fragmented DNA. At least 500 sperm were observed to obtain the number of unfragmented and fragmented DNA. Sperm with unfragmented DNA showed either large- or medium-sized halos, while those with fragmented DNA showed either small halo or no halo. The DFI was classified as (1) good (0-15\%); (2) moderate (>15-25\%); and (3) severe (>25\%).

\section{Statistical analysis}

This study analyzes: (1) Demographic description of subjects having risk factors of infertility, (2) the influence of demographic and subject's comorbid on DFI using Chi-square, to identify factors other than surgery that affect the incidence of infertility, (3) the effect of the sperm quality at pre-processing and after 
5 min SU, (4) the effect of the sperm quality at preprocessing and after $10 \mathrm{~min}$ SU, and (5) the effect of sperm quality at 5 and $10 \mathrm{~min}$ SU. Statistical analysis was performed using SPSS version 16.0 for Windows (Chicago, IL, USA) with a significance level of $5 \%(0.05)$ for absolute and $10 \%(0.1)$ for relative.

\section{Ethical approval}

This study received ethical approval from Stella Maris Women's and Children's Hospital, Medan, Indonesia, with ethical number Ethical Approval No. 496-1/Dir/RSIA.SM/VI/2020.

\section{Results}

Fifty subjects met the inclusion criteria and studied until the final analysis. The routine semen and sperm DNA fragmentation analysis were conducted at Halim Fertility Center, Stella Maris Women's and Children's Hospital, Medan, Indonesia, from June 2020 to October 2020.

From 50 subjects, $24(30.75)$ subjects are below 35 years old and 26 (38.77) subjects are older than 35 years old, with the distribution of one subject with underweight body mass index (BMI), 16 subjects with normoweight BMI, 22 subjects with overweight $\mathrm{BMI}, 11$ subject with obese BMI, 15 subjects with the history of smoking, and 4 subjects with the history of consuming alcohol (Table 1).

Table 1: Patients characteristics

\begin{tabular}{lll}
\hline Characteristic & Total $(\mathrm{n}=50)$ & Mean \\
\hline Age, $\mathrm{n}(\%)$ & & \\
$\quad<35$ year & $24(48)$ & 30.75 \\
$\quad>35$ year & $26(52)$ & 38.77 \\
BMl, n (\%) & $1(2)$ & 17.76 \\
$\quad$ Underweight & $16(32)$ & 23.04 \\
$\quad$ Normoweight & $22(44)$ & 27.42 \\
$\quad$ Overweight & $11(22)$ & 32.93 \\
$\quad$ Obesity & $15(30)$ & - \\
Smoking, n (\%) & $35(70)$ & - \\
Yes & & - \\
No & $4(8)$ & - \\
Alcohol, n (\%) & $46(92)$ & \\
Yes & & \\
No & &
\end{tabular}

It was found that age, BMI, smoking, and alcohol consumption did not have a significant effect to DFI (Table 2).

Table 2: The relationship between patients characteristics and DFI

\begin{tabular}{llll}
\hline Characteristic $(\mathrm{n}=50)$ & OR & $\mathrm{DFI}, \mathrm{n}=50$ & $\mathrm{p}$ \\
\cline { 2 - 4 } & & $\mathrm{Cl} 95 \%$ & 0.66 \\
\hline Age & 0.77 & $0.75-0.76$ & 0.26 \\
BMI & 0.61 & $0.34-0.36$ & 0.77 \\
Smoking & 0.60 & $0.88-0.90$ & 0.40 \\
Alcohol & 0.69 & $0.45-0.47$ &
\end{tabular}

Statistical significant differences were found in comparison of sperm quality at pre-processing and after 5 min SU $(p<0.05)$, where sperm concentration, motility, and progressive motility were found higher after $5 \mathrm{~min}$ SU, while the DFI was lower after $5 \mathrm{~min}$ SU (Table 3).

Table 3: Comparison of sperm quality at pre-processing and after $5 \mathrm{~min}$ SU

\begin{tabular}{llll}
\hline Parameters & Pre-processing & 5 min SU & $\mathrm{p}$ \\
\hline C & $28.57(24.85-32.33)^{*}$ & $33.87(27.78-39.79)^{*}$ & 0.018 \\
M & $47.40(43.78-51.08)^{*}$ & $97.97(97.66-98.20)^{*}$ & 0.001 \\
PM & $2.42(0-24)^{\star *}$ & $13.68(0-41)^{\star *}$ & 0.001 \\
DFI & $16.12(13.48-19.04)^{\star}$ & $5.30(3.82-6.98)^{\star}$ & 0.001 \\
\hline C: Concentration, M: Motility, PM: Progressive motility, DFI: DNA fragmentation index, $\left(^{*}\right):$ Paired t-test, $\left(^{\star \star}\right):$ \\
Wilcoxon, SU: Swim-up.
\end{tabular}

Significant differences were also found in comparison of sperm quality at pre-processing and after 10 min SU $(p<0.05)$, where sperm concentration, motility, and progressive motility were found higher after $10 \mathrm{~min}$ SU, while the DFI was lower after $10 \mathrm{~min}$ SU (Table 4).

Table 4: Comparison of sperm quality at pre-processing and 10 minutes SU

\begin{tabular}{llll}
\hline Parameters & Pre-processing & 10 min SU & $\mathrm{p}$ \\
\hline $\mathrm{C}$ & $28.57(24.68-32.65)^{\star}$ & $42.80(35.36-51.09)^{*}$ & 0.001 \\
M & $47.40(43.78-50.90)^{\star}$ & $98.04(97.86-98.20)^{*}$ & 0.001 \\
PM & $2.42(0-24)^{\star *}$ & $18.38(0-54)^{\star *}$ & 0.001 \\
DFI & $16.12(4-47)^{\star *}$ & $7.84(1-25)^{\star *}$ & 0.001 \\
\hline C: Concentration, M: Motility, PM: Progressive motility, DFI: DNA fragmentation index, $\left.{ }^{\star}\right)$ : Paired t-test, \\
${ }^{* \star}$ : Wilcoxon, SU: Swim-up.
\end{tabular}

Significant differences in sperm concentration, progressive motility, and DFI were found between the sperm quality after 5 and 10 min SU $(p<0.05)$, where sperm concentration, progressive motility, and DFI were higher after 10 min centrifugation SU, but no significant differences were found in terms of sperm motility between 5 and $10 \mathrm{~min} S U(p>0.05)$ (Table 5).

Table 5: Comparison of sperm quality after $5 \mathrm{~min}$ and $10 \mathrm{~min}$ SU

\begin{tabular}{|c|c|c|c|}
\hline Parameters & $5 \min \mathrm{SU}$ & $10 \mathrm{~min} S U$ & $\mathrm{p}$ \\
\hline $\mathrm{C}$ & $33.86(28.18-39.88)^{*}$ & $42.80(35.81-49.59)^{*}$ & 0.001 \\
\hline M & $97.96(94-99)^{\star \star}$ & $98.04(97-99)^{\star \star}$ & 0.599 \\
\hline PM & $13.68(0-41)^{\star \star}$ & $18.38(0-54)^{\star \star}$ & 0.001 \\
\hline DFI & $5.30(0-23)^{\star \star}$ & $7.84(1-25)^{\star \star}$ & 0.001 \\
\hline
\end{tabular}
Wilcoxon, SU: Swim-up.

\section{Discussion}

The infertility status of a male patient was formerly determined by a routine semen analysis, which included volume, $\mathrm{pH}$, concentration, motility, and the morphology of the sperm. Recently, studies have shown that DNA integrity can be used as a predictor of fertility and it has been one of the important parameters to determine the sperm quality because fragmented DNA is known to be found more in patients with abnormal semen analysis [8]. DNA integrity is important for sperm to carry out the function of germ cells and for the early stage of embryo development [9]. Although studies have shown that sperm with fragmented DNA is able to fertilized the oocytes, it may affect the early and cleavage stage of embryo development, embryo quality, as well as recurrent pregnancy loss after IVF and intracytoplasmic sperm injection [8], [9], [10]. 
Assisted reproductive technologies (ARTs), such as intrauterine insemination (IUI) and IVF, have been used to treat couples with infertility, where semen parameters are known to play important roles in successful ART cycles. The sperm count after semen processing is considered to be a determining factor for a successful ART cycle, particularly IUI, though it is still controversial [11]. Some sperm processing methods have been established to retrieve more sperm with better quality such as density gradient, SU, and washing [9], [12]. The present study stated that semen samples which are processed using the SU method, either after 5 and $10 \mathrm{~min}$ centrifugation, showed significantly better results $(p<0.05)$ in terms of sperm concentration, total motility, and progressive motility, as well as DFI than fresh semen samples. Our study showed similar results as the past studies, where semen samples processed using the SU method yielded less sperm with fragmented DNA and ROS. Furthermore, this method may also yield more motile sperm than fresh samples [2], [9], [13], [14].

The present study showed that semen samples processed with 10 min SU method gave a significantly better results in terms of sperm concentration and progressive motility $(p<0.05)$. However, the DFI of the sperm centrifuged for $10 \mathrm{~min}$ was found higher than those centrifuged for $5 \mathrm{~min}(p<0.05)$. This result was in conjunction to the study where sperm preparation method involves centrifugation process to retrieve the pellets. The centrifugation process itself is known to increase the production of ROS which may lead to sperm DNA damage [15].

However, in contrast to our findings, some past studies stated that the sperm concentration after processing is lower than those before processing [15], [16], [17]. The difference in our results might be caused by the sperm retrieval rate after SU varies depending on the semen quality as well as the modification of the preparation technique used. Besides, our inclusion criteria were different from other studies, where we included the oligoasthenoteratozoospermic samples, while others only included the normozoospermic samples. Another suggestion for the low sperm retrieval rate after processing was the declining of sperm ability to migrate from the dense pellet toward the culture media layered above it. The proportion of migrating sperm might differ, depending on the temperature, the interphase between two liquids, and the duration of incubation [16].

\section{Conclusion}

The sperm concentration, total motility, progressive motility, and DFI processed with SU method yield better results than fresh samples.
Moreover, a longer centrifugation time during semen processing may affect the DNA integrity of the sperm. The limitations of this study are the small number of samples and the large gap in sperm concentration as the inclusion criteria. Hence, in future studies, we may need a larger number of samples and a stricter inclusion criteria to validate the result better.

\section{References}

1. Sun TC, Zhang Y, Li HT, Liu XM, Yi DX, Tian L, et al. Sperm DNA fragmentation index, as measured by sperm chromatin dispersion, might not predict assisted reproductive outcome. Taiwan J Obstet Gynecol. 2018:57(4):493-8. https://doi. org/10.1016/j.tjog.2018.06.003

PMid:30122567

2. Al-Marayaty SS, Saeed GT, I. Al-Ahmed H. Effect of swim up techniques on sperm motility and DNA integrity versus unprepared semen. J Fac Med. 2017;59(2):151-5. https://doi. org/10.32007/med.1936/jfacmedbagdad.v59i2.10

3. Punjabi U, Van Mulders $H$, Goovaerts I, Peeters $K$, Clasen $\mathrm{K}$, Janssens $\mathrm{P}$, et al. Sperm DNA fragmentation in the total and vital fractions before and after density gradient centrifugation: Significance in male fertility diagnosis. Clin Biochem. 2018;62(1):47-54. https://doi.org/10.1016/j. clinbiochem.2018.05.011 PMid:29792832

4. Halim B. The association between sperm DNA fragmentation and idiopathic early recurrent pregnancy loss. KnE Med. 2016;1(1):1-9. https://doi.org/10.18502/kme.v1i1.537

5. Lestari SW, Lestari SH, Pujianto DA. Sperm quality after swim up and density gradient centrifugation sperm preparation with supplementation of alpha lipoic acid (ALA): A preliminary study. AIP Conf Proc. 2018;2018:1933. https://doi. org/10.1063/1.5023962

6. Xue X, Wang WS, Shi JZ, Zhang SL, Zhao WQ, Shi WH, et al Efficacy of swim-up versus density gradient centrifugation in improving sperm deformity rate and DNA fragmentation index in semen samples from teratozoospermic patients. J Assist Reprod Genet. 2014;31(9):1161-6. https://doi.org/10.1007/ s10815-014-0287-z

PMid:25015033

7. Younglai EV, Holt D, Brown P, Jurisicova A, Casper RF. Sperm swim-up techniques and DNA fragmentation. Hum Reprod. 2001;16(9):1950-3. https://doi.org/10.1093/humrep/16.9.1950 PMid: 11527903

8. Park YS, Lee SH, Choi HW, Lee HS, Lee JS, Seo JT. Abnormal human sperm parameters contribute to sperm DNA fragmentation in men with varicocele. World J Mens Health. 2018;36(3):239-47. https://doi.org/10.5534/wjmh.180014 PMid:30079641

9. Zhao F, Yang Q, Shi S, Luo X, Sun Y. Semen preparation methods and sperm telomere length: Density gradient centrifugation versus the swim up procedure. Sci Rep. 2016;6(1):39051. https://doi.org/10.1038/srep39051 PMid:27958357

10. Hamilton TRDS, Assumpcã̃ MEODÁ. Sperm DNA Fragmentation: Causes and Identification. Zygote. Assumpcąõ MEODÁ; 2019. https://doi.org/10.1017/s0967199419000595

11. Koyun Ok E, Erbil Doğan Ö, Emre Okyay R, Gülekli B. The effect of post-wash total progressive motile sperm count and semen 
volume on pregnancy outcomes in intrauterine insemination cycles: A retrospective study. J Turk Ger Gynecol Assoc. 2013;14(3):142-5. https://doi.org/10.5152/jtgga.2013.52280

PMid:24592093

12. Repalle D, Chittawar PB, Bhandari S, Joshi G, Paranjape M, Joshi C. Does centrifugation and semen processing with swim up at $37^{\circ} \mathrm{C}$ yield sperm with better DNA integrity compared to centrifugation and processing at room temperature? J Hum Reprod Sci. 2013;6(1):23-6. https://doi. org/10.4103/0974-1208.112375

PMid:23869146

13. Nabi A, Khalili MA, Halvaei I, Roodbari F. Prolonged incubation of processed human spermatozoa will increase DNA fragmentation. Andrologia. 2014;46(4):374-9. https://doi. org/10.1111/and.12088

PMid:24689689

14. Ghaleno LR, Valojerdi MR, Janzamin E, Chehrazi M, Sharbatoghli M, Yazdi RS. Evaluation of conventional semen parameters, intracellular reactive oxygen species, DNA fragmentation and dysfunction of mitochondrial membrane potential after semen preparation techniques: A flow cytometric study. Arch Gynecol Obstet. 2014;289(1):173-80. https://doi. org/10.1007/s00404-013-2946-1

PMid:23846620

15. Ricci G, Perticarari S, Boscolo R, Montico M, Guaschino S, Presani G. Semen preparation methods and sperm apoptosis: Swim-up versus gradient-density centrifugation technique. Fertil Steril. 2009;91(2):632-8. https://doi.org/10.1016/j. fertnstert.2007.11.068

PMid: 18206147

16. Adiga SK, Kumar P. Andrology: Influence of swim-up method on the recovery of spermatozoa from different types of semen samples. J Assist Reprod Genet. 2001;18(3):160-4.

PMid:11411432

17. Fácio CL, Previato LF, Machado-Paula LA, Matheus PC, Filho EA. Comparison of two sperm processing techniques for low complexity assisted fertilization: Sperm washing followed by swim-up and discontinuous density gradient centrifugation. J Bras Reprod Assist. 2016;20(4):206-11. https://doi. org/10.5935/1518-0557.20160040

PMid:28050954 